\title{
The analysis of a four-span skew bridge using an electrical analogue computer
}

\author{
by \\ C. T. Harnden, M.Sc.
}

and

K. R. Rushton, B.Sc., Ph.D., A.M.I.C.E.

Professor O. C. Zienkiewicz and Mr I. P. King (Department of Civil Engineering, University of Wales) wrote that the Authors had produced an interesting application of electrical analogue networks to a complex stress analysis problem.

62. The digital computer presented an extremely versatile and economic approach to such problems, and programs had been developed to apply the finite element method with triangular elements ${ }^{6.7}$ to plate bending problems such as skew bridges. One such program allowed for many complexities, such as:

(a) variable thickness plates;

(b) orthotropic structure;

(c) irregular geometry;

(d) elastic column supports;

(e) additional girders in any direction.

The program had been successfully tested against exact and experimental solutions on a number of occasions and appeared to present a more versatile and economical procedure than that given in the Paper.

63. For comparison an analysis had been made of the simplified example described by the Authors, the case with uniform load $q$ over the whole area being chosen. To confirm convergence of the solution three mesh layouts were used, (A), (B), (C) in Fig. 13. The similarity of the automatically plotted contours of $M_{y}$ for (A) and (C) shown in Fig. 14, demonstrated the good convergence of the solution. For a typical case the total time involved in preparation of data, using automatic mesh generation, was less than three hours. The ICT 1905 computer took less than 15 min for case (B) and additional load distributions added approximately one minute per load case. This order of subdivision was probably adequate for most practical cases.

64. Fig. 15 showed section plots of displacement along an edge and moment along the centre line. The finite element results showed reasonable agreement with the Authors on the centre line moment (when allowance was made for the averaging procedure of automatic plotting). There was a discrepancy in displacement along the edge. The difference was however not much more than the $5 \%$ indicated by the Authors as the margin of error.

65. Clearly it was difficult to assess the accuracy of two approximate methods on such a complex structure where no absolute standard existed.

66. The distribution of support reactions obtained by the finite element method showed a considerable discrepancy from that given by the Authors (Fig. 16). The reactions obtained from other finite element bridge analyses had shown good correlation with exact and experimental values. It was particularly noticeable that the Authors' reactions did not show the well known concentration and reversal at reentrant corners; the finite element results seemed more realistic. 

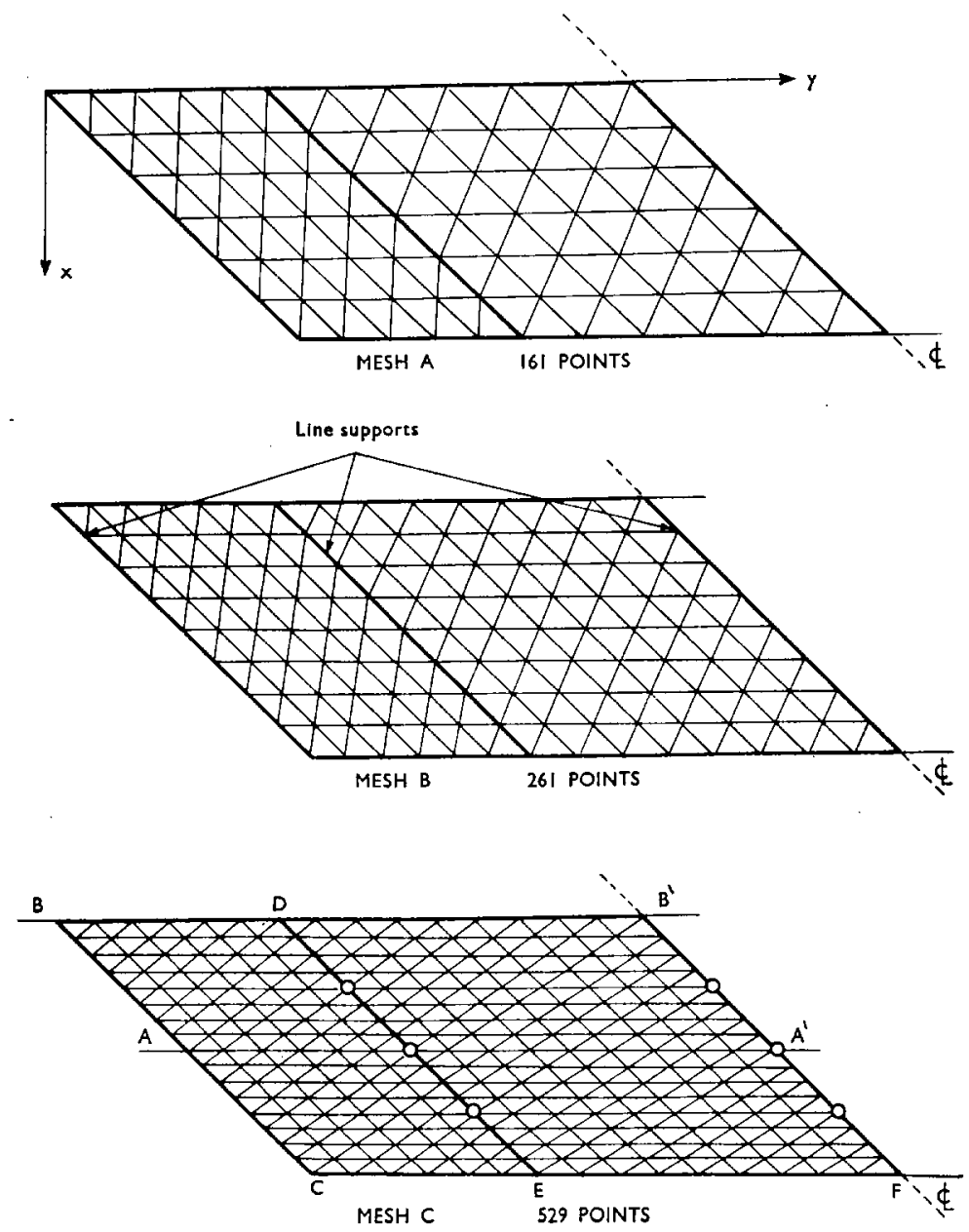

Fig. 13: Triangular MESH LAYOUTS (HALF BRIDGE ShOWN)

67. The Authors indicated that real bridges were commonly supported on columns and, as previously mentioned, such a structure was again readily analysed in finite element programs. The resulting moments for the same four-span skew bridge supported on columns over its interior spans (Fig. 13) were shown in Fig. 17. Naturally the infinite moments over the columns were shown only by trends, but the change in displacements and moments were quite considerable. A finer mesh around the columns would give more detailed values for moments; in this case it would be necessary to assign a finite area to the column tops.

Mr C. M. McMillan (Messrs Ove Arup and Partners) wrote that he had found the Paper of interest, especially as he had been concerned with skew slab analysis by finite difference methods, in research at the University of the Witwatersrand, Johannesburg. 

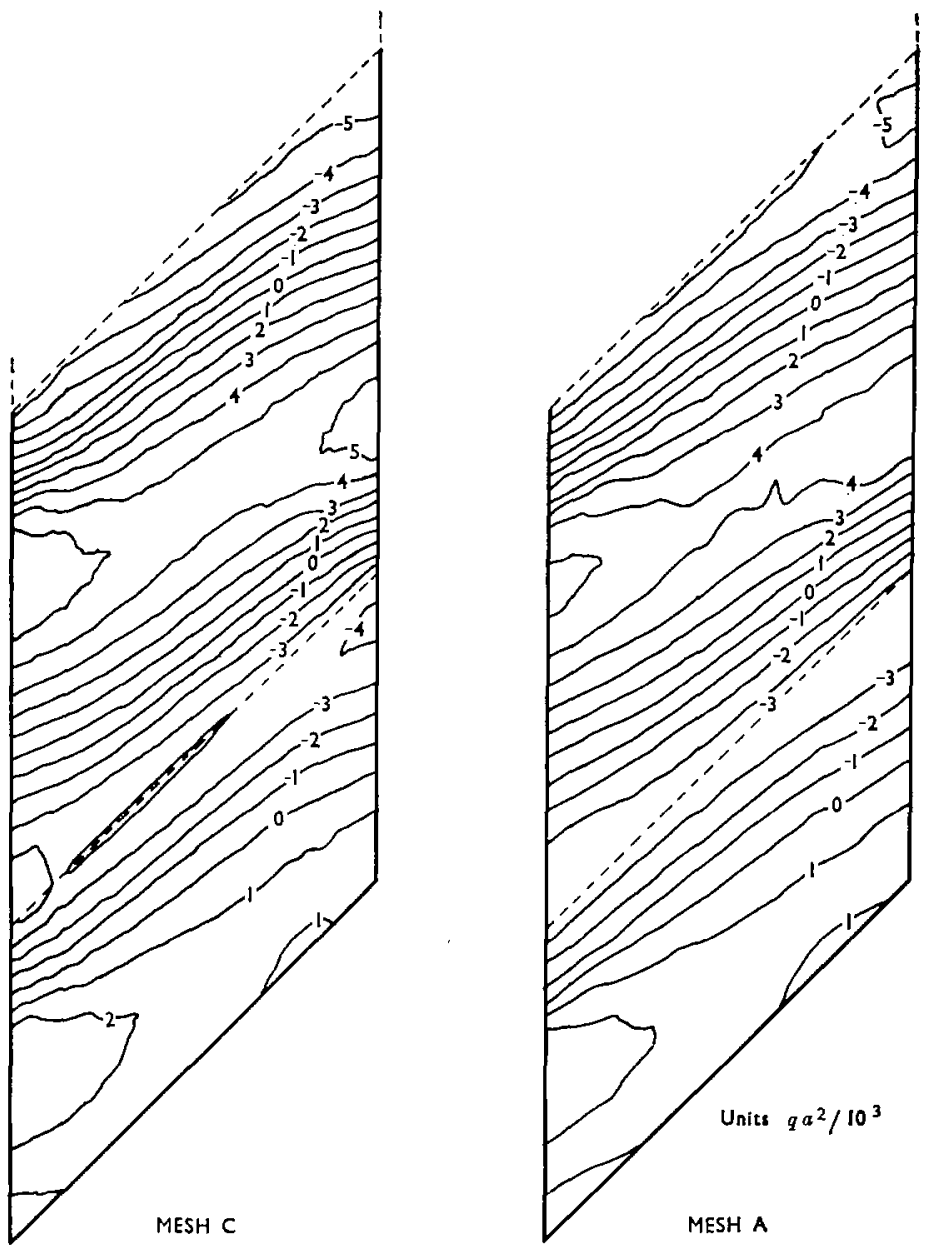

Fig. 14: Contours of MOMENT $M_{y}$

He wished to draw some comparisons with other methods of analysing the structure under consideration, and to ask for further details on a few points.

69. Firstly, it appeared that the network used was excessively fine, and the accuracy of the results bore this out. It was wondered whether the Authors would recommend a coarser network for future analyses, and whether significant savings in time and apparatus could be achieved in this way.

70. The rectangular network had certain disadvantages when compared with the skew network proposed by Jensen. ${ }^{8}$ For nodes to be located on the support lines, a chosen mesh spacing in one direction fixed the spacing in the other direction. The problem under consideration was ideal, in that the $45^{\circ}$ skew angle made the spacings equal in the $x$ and $y$ directons. In general, if the spacing in the $x$-direction were $h$, and the angle of skew were $\theta$, the spacing in the $y$-direction would be $h \tan \theta$. For 


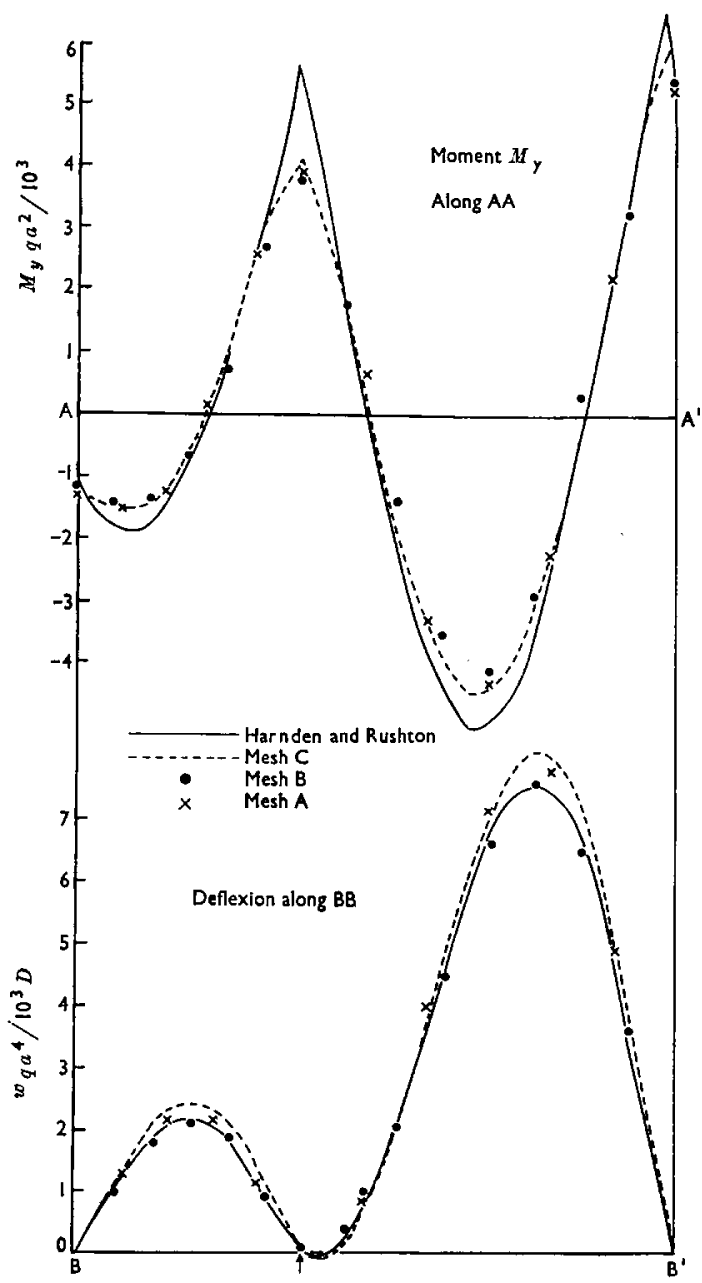

Fig. 15: SeCtion PLOTS

practical angles of skew one might therefore be obliged to accept a mesh interval in one direction of double that in the other, regardless of the span-to-width ratio of the slab.

71. Furthermore, such a network involved tailoring the dimensions of the slab in one direction to be a multiple of the mesh spacing chosen in the other direction.

72. There were many advantages in using a digital computer to solve the finite difference equations. An $8 \times 8$ network on each span would be adequate. ${ }^{9.10}$ By making use of symmetry only two spans needed to be considered. Unsymmetrical loads could then be dealt with automatically in the program by superposition of symmetrical and anti-symmetrical components. By storing only the terms in the non-zero band of the matrix to the right of the main diagonal, the problem would 
necessitate the storage of a $126 \times 21$ matrix. This would not require an excessively large computer. By sophisticated programming and the use of backing store, considerably larger structures could be accommodated, although the number of equations to be solved would eventually make the method impractical.

73. The capital outlay on such a program would not be excessive, and might compare favourably with the expenditure on setting up the electrical analogue. After that the digital computer program would be very efficient for dealing with numerous different loading cases. The Writer wondered whether the Authors could put figures to the development expenditure, and the time to achieve balanced conditions for any particular load case.

74. The program visualized could be generalized without difficulty to deal with stiffening beams along the unsupported edges, or an orthotropic slab. It would be interesting to know what these two effects involved in the electrical analogue.

75. The Writer was particularly interested to see that the problem of finding distributions of support reactions had been tackled. He had had great difficulty in obtaining reliable results for these, for single span slabs. Internal support points presented no trouble: the Authors' equation (9) or its equivalent for the skew mesh would presumably give a reliable result by either method. At a simply supported edge, however, $M_{0}$ would be zero, being a moment on the edge, while two of points 1,2 , 3 and 4 would lie outside the slab boundary. He wished to know what additional assumptions, if any, were made in calculating the reactions on these edges. Similar problems arose at edge points on the internal supports, while at the four corner points a reliable estimate of the intensity of reaction seemed impossible.

76. The Authors referred to the adaptability of the electrical analogue ' to simulate the plates of various shapes and the different boundary conditions arising in skew bridge structures'. In the Writer's opinion both the numerical method and the analogue suffered from the same drawback here. A change in the geometry or boundary conditions required a modified programme or a reconstructed circuit.

77. To overcome this two other methods of analysis seemed to offer great advantages. One was the commonly used equivalent grillage method, in which the slab was replaced by an equivalent grid of beams, to which bending and torsional rigidities were assigned. Any standard grid analysis program could then be used, data preparation was simple, and the boundary conditions presented no problems. The method was mathematically justifiable, and gave reliable results in practice.

78. Still more promising was the method of finite elements, which was as yet not widely used in civil engineering. It offered all the advantages of generality and simplicity of application, but in addition was likely to give more reliable results for a given fineness of grid.

79. For both methods general programs were available to handle slabs of any shape, with arbitrary support conditions including elastic restraints, and the only limitation was the size of the resulting stiffness matrix. With the increasing availability of computer services to design engineers, such methods were likely to become more and more commonly used.

Dr W. M. Jenkins and Mr J. M. Siddall (University of Bradford) wrote that the analysis of continuous skew bridges was of considerable importance and the Authors were to be congratulated on carrying out a detailed examination of the use of the analogue computer in obtaining a solution. The iterative procedure outlined involved a certain amount of trial and error, for example in setting the applied line moments simulating a vertical support, $\S \S 21,22,23$, and a good initial estimate of boundary moments and deflexions was required for satisfactory application of the method in $\$ 44$. Analogue computations of this nature and complexity require a skilled technique and it was doubtful if they would ever become a widely accepted design method. A digital approach to the computation was more promising in that once a digital computer program had been produced the analysis of any bridge was a routine matter. 
81. A computer program suitable for ICT 1900 and ATLAS computers had been prepared at the University of Bradford. In order to make the program as useful as possible certain parameters had been written in allowing solutions to be produced for a wide range of bridges.

82. The plan of the bridge was assumed to be a parallelogram with angle of skew $\delta$ (Fig. 18). The length of the bridge was divided into $M$ divisions and the width - into $N$ divisions. All $M$ divisions longitudinally might be of different lengths which allowed complete freedom in representing different spans in a continuous system. In choosing the $N$ divisions across the bridge the program permitted the specification of two different widths. The bridge deck was then represented by an array of $M \times N$ finite elements and a stiffness matrix for a typical parallelogram element had been produced.

83. The program provided for longitudinal and transverse beams along any of the nodal lines. A facility existed in the data for specifying changes in thickness of deck slab should these occur. For a uniform thickness slab and uniform element dimensions the computer assembled only one element stiffness matrix. The structure stiffness matrix was assembled in half symmetrical band form and solved by elimination for the nodal displacements.

84. The data consisted of the dimensions of the bridge, the parameters $M, N$ and $\delta$, and details of the beams if present. Vertical support might be provided at any of the nodal points or along lines connecting nodes. In the latter case this was achieved by imposing zero values on $w$ and $\theta_{y}$ along the line support.

85. The bridge example of the Authors had been analysed using the program for an overall uniformly distributed load in order to obtain a comparison and the results obtained pertaining to section AA in the figure were shown plotted. It was seen that the results were in good agreement for $M_{y}$ and $M_{x y}$. The differences in the maximum values of $w$ and $M_{x}$ were $8 \%$ and $17 \%$ respectively, the results from the digital solution being higher than those from the analogue solution for $M_{x}$ and lower for $w$. In the calculation $M$ was taken as 32 and $N$ as 7 . A finer subdivision of the bridge was possible but was unlikely to be necessary in the majority of designs. The time for compiling the program and running was approximately one minute on ATLAS using 38000 words storage.

Professor C. B. Wilby and Mr I. Khwaja (University of Bradford) wrote that the linear simultaneous equations obtained from finite difference approximations to the governing biharmonic equation had been solved by using an electrical analogy. These equations could alternatively be solved using a library program for an electronic digital computer. It seemed difficult to. imagine a case where the designer would prefer to use the electrical analogy.

87. The paper in 1933 by Hay ${ }^{11}$ used an electrical analogy for solving simultaneous equations, but at that date electronic digital computers were not available and electronic desk calculating machines were not invented, electrical desk calculating machines were rare, if invented, and hand-operated desk calculating machines were not very common in design offices. At that date, therefore, the electrical analogy was perhaps a very useful method, but for many reasons little advantage was taken of the method in practice.

88. In the book by Wilby and Bellamy ${ }^{12}$ the electronic analogue computer method had advantages over the electronic digital computer in that it assisted in the design of a shell as opposed to the analysis only of a shell. It was also relatively easier to deal with more complex theories. ${ }^{13}$

89. They mentioned, in passing, that some engineers had mistakenly gathered the impression that the analogue computer was not accurate enough for shell problems. This was not so as anyone could see from a detailed study of reference (2). The answers were as accurate as required by the designer for any shell problem as far as the Writers were aware - the method was different from the normal method used on 
a desk calculator or digital computer, in that it cut out the need to solve large numbers of simultaneous equations and it was the solution of these equations which needed the high accuracy of the calculating machine or digital computer.

90. In what circumstances would the Authors consider that the practising designer should use the electrical analogy as opposed to the desk calculator or digital computer? Or do they consider that the electrical analogy can have extra advantages over other methods?

91. The number of nodal points and hence the number of simultaneous equations required to be solved would depend on the dimensions of the slab and the size of finite difference grid.

92. In the example given by the Authors the grid size was such that there were 1000 nodal points, giving 1000 equations. It would be impossible to tackle such a large number of equations on a digital computer with a reasonable storage. However, most of the terms in these equations were zero (for the biharmonic equation, the number of non-zero terms in each finite difference equation would be only 13), and the equations could be written in the form of a narrow diagonal band matrix requiring a comparatively small computer store. Library programs to deal with such matrices were available and it would be found that by using these programs a moderately sized digital computer could handle a reasonable number of such equations.

93. It would seem that a problem resulting in a modest number of simultaneous equations would be more economically designed with an electronic digital computer (posting the problem to a computer centre if necessary) or with an electronic, electric, or hand, desk calculating machine, rather than by electrical analogy.

94. A problem resulting in numerous simultaneous equations would appear to be much more economically solved by use of the electronic digital computer.

95. No matter how remote a design office might be, it could always make use of a computer centre by post and this was a speedy and inexpensive procedure compared with the expense and time involved in designing and making electrical analogies.

Mr F. Sawko and Mr R. J. Cope (Department of Civil Engineering, Leeds University) wrote that they had read with great interest the Authors' Paper, and were most impressed by the extreme care that has gone into setting up of the analogue circuit for this complex structure and by the excellent presentation of the results.

97. The Writers agreed that no rigorous solution of the differential equation for bending of plates with skew supports existed. Furthermore, in practical bridge structures orthotropy, edge stiffening and non-parallel continuous or discrete supports often existed, rendering any exact continuum solution inconceivable. Solution was only practicable using numerical methods, and the Authors had adopted the finite difference approach based on a square mesh, utilizing the analogue for solving the very large sets of equations. The Writers would like to present an alternative in the form of the finite element approach, which they believed offered many advantages, especially in the initial design stages when many different configurations had to be analysed before the design was finalized.

98. Zienkiewicz and Cheung ${ }^{14}$ have demonstrated the power, flexibility and accuracy of the rectangular flexural element, when a deformation function in the form of a cartesian polynomial was adopted. The Writers had demonstrated the accuracy of the method when applied to right orthotropic bridge decks. ${ }^{15}$ Studies on skew bridge decks carried out at Leeds ${ }^{16}$ had revealed that it would be desirable to retain the basic rectangular element, and this led to the development now described.

99. If a rectangular mesh was considered to be parallel to the free boundaries, triangular elements were formed along the end abutments. The Authors modified the basic stiffness matrix of a rectangular element by constraining the hypotenuse to rotate about a line axis thus producing a single line support. The deformation of the element thus derived was compatible with the rectangular elements, and the whole procedure had the advantage of good accuracy for a given mesh size. ${ }^{16}$ This was 


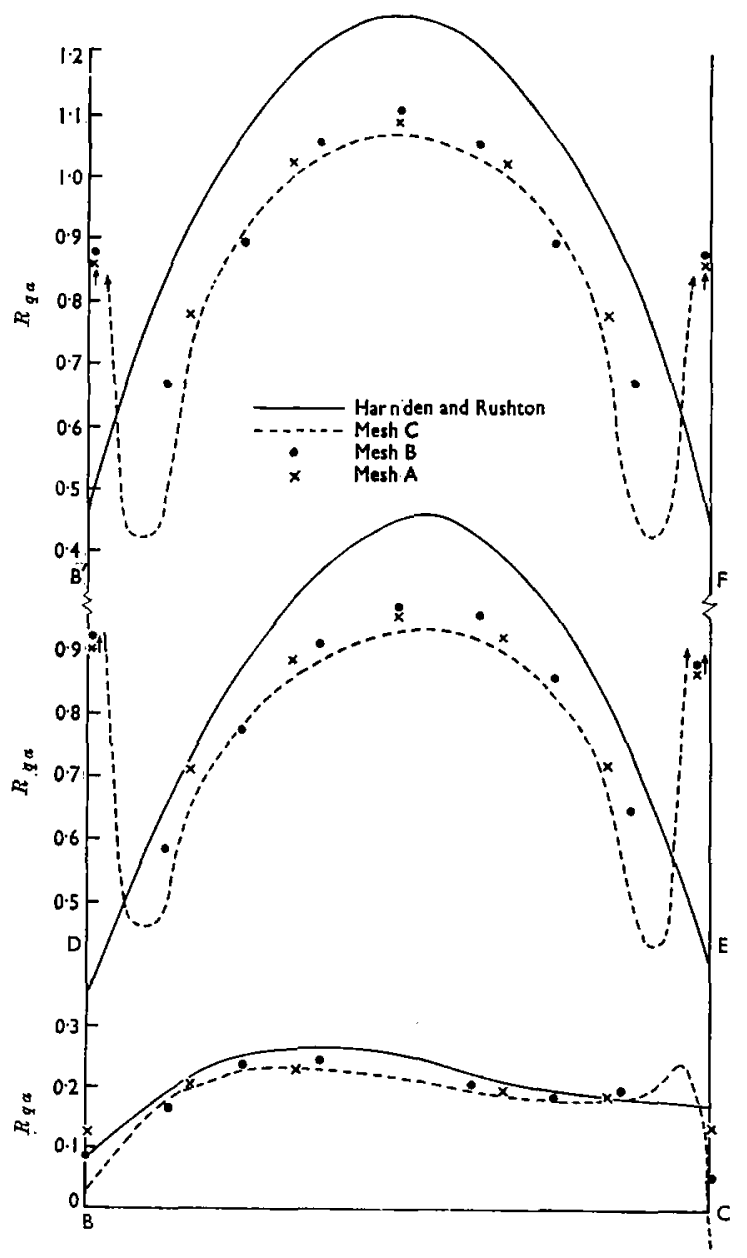

FIG. 16 (above): REACTION ALONG SUPPORT LINES

Fig. 17 (top, right): Section MOMENT Alono AA (Mesh C)

Fig. 18 (right): Comparisons of aNAlogue aNd digital solutions along LiNE AA FOR BRIDGE CARRYING UNIFORMLY DISTRIBUTED LOAD OVERALL 

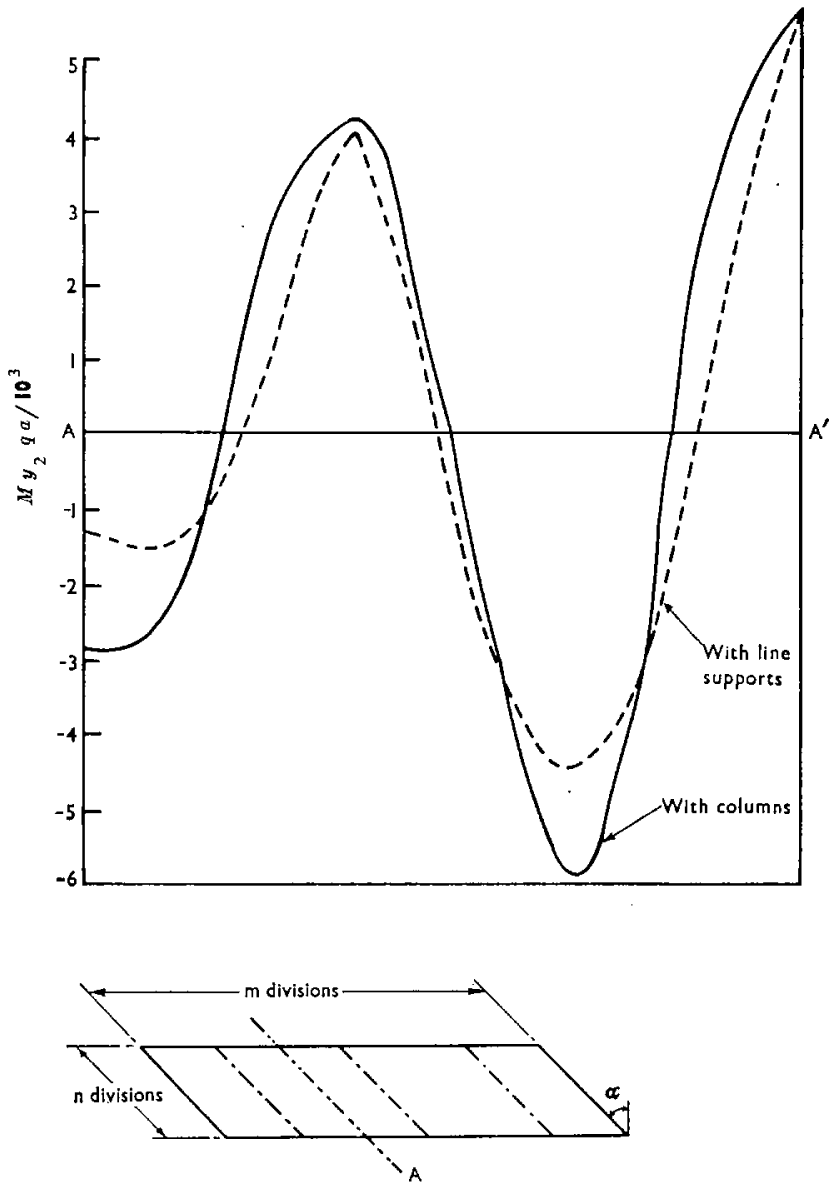

PLAN OF BRIDGE
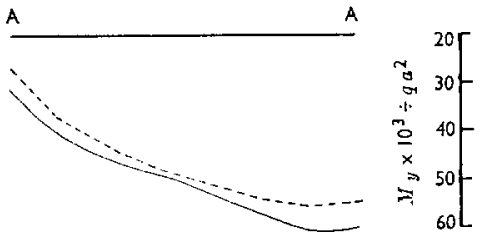

A
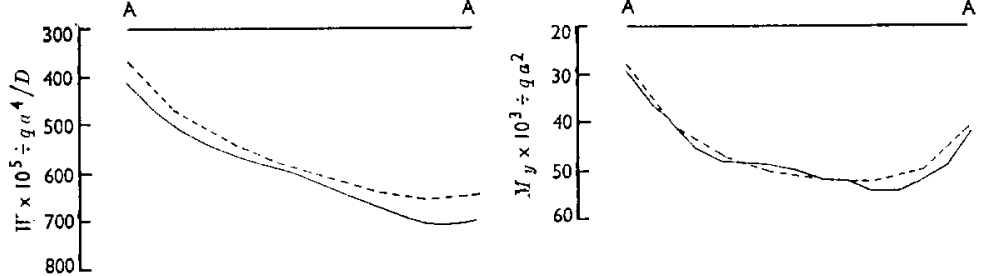

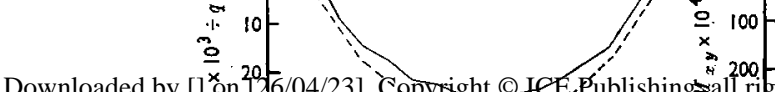

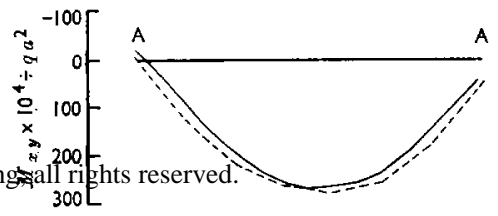



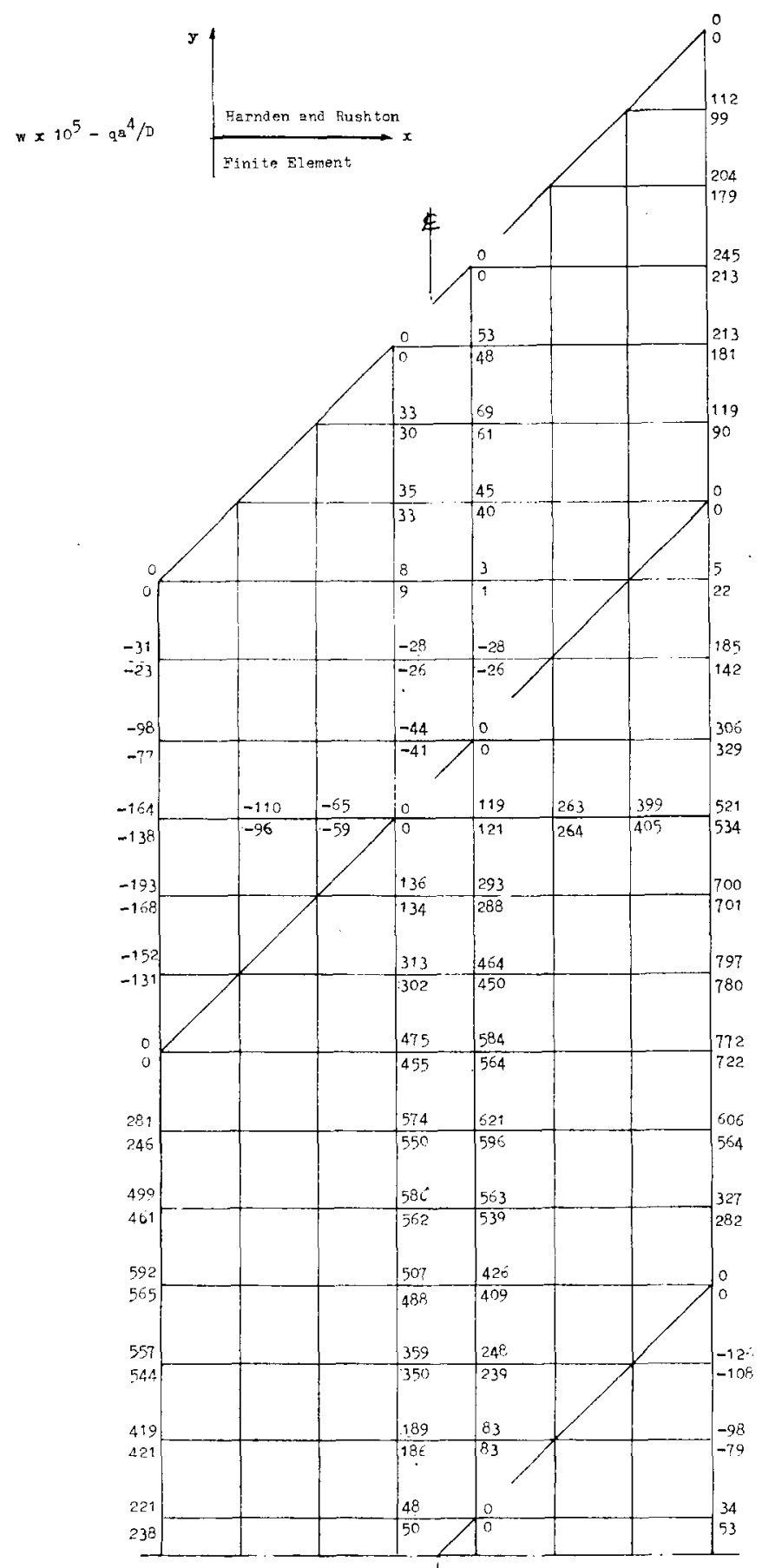

Fig. 19: Deflexions FOR SKEW BRIDGE CARRYING A UNIFORM LOAD

Downloaded by [] on [26/04/23]. Copyright @ ICE Püblishing, all rights reserved. 


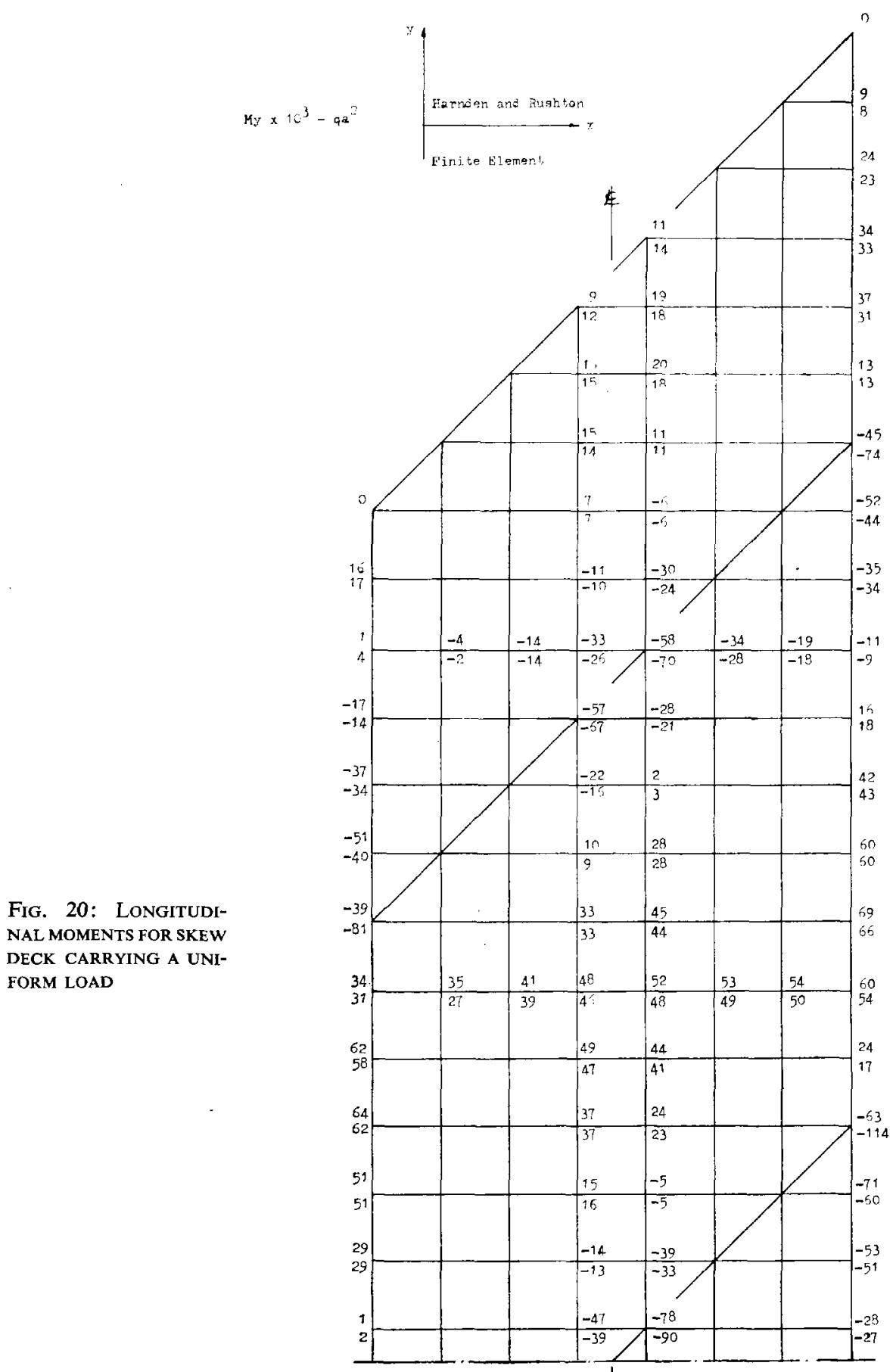

Downloaded by [] on [26/04/23]. Copyright @ ICE Publishing, all rights reserved. 


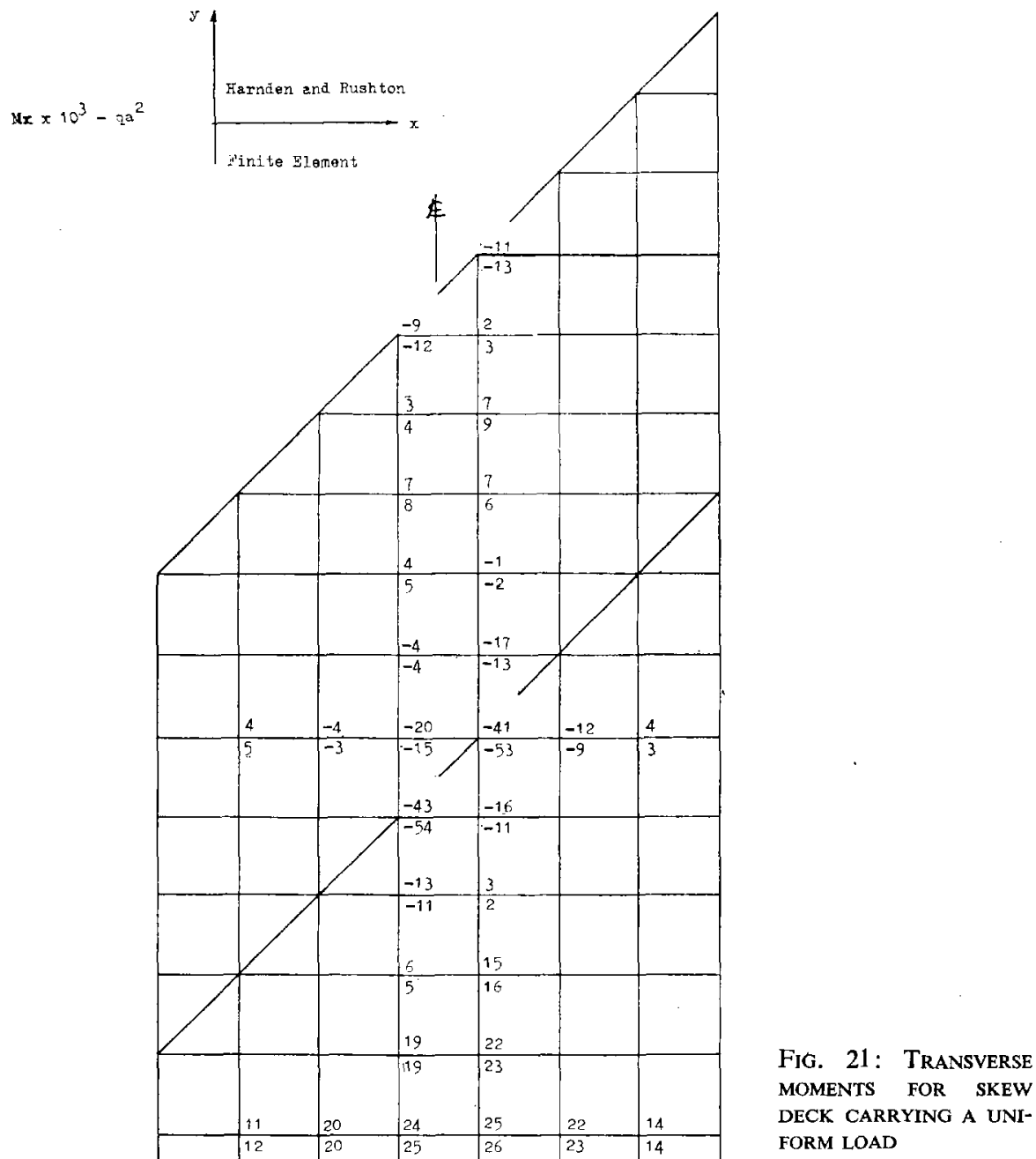

Downloaded by [] on [26/04/23]. Copyright @ ICE Publishing, all rights reserved. 


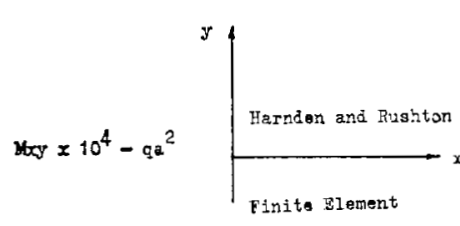

Fig. 22: TWISTING MOMENTS FOR SKEW DECK CARRYING A UNIFORM LOAD

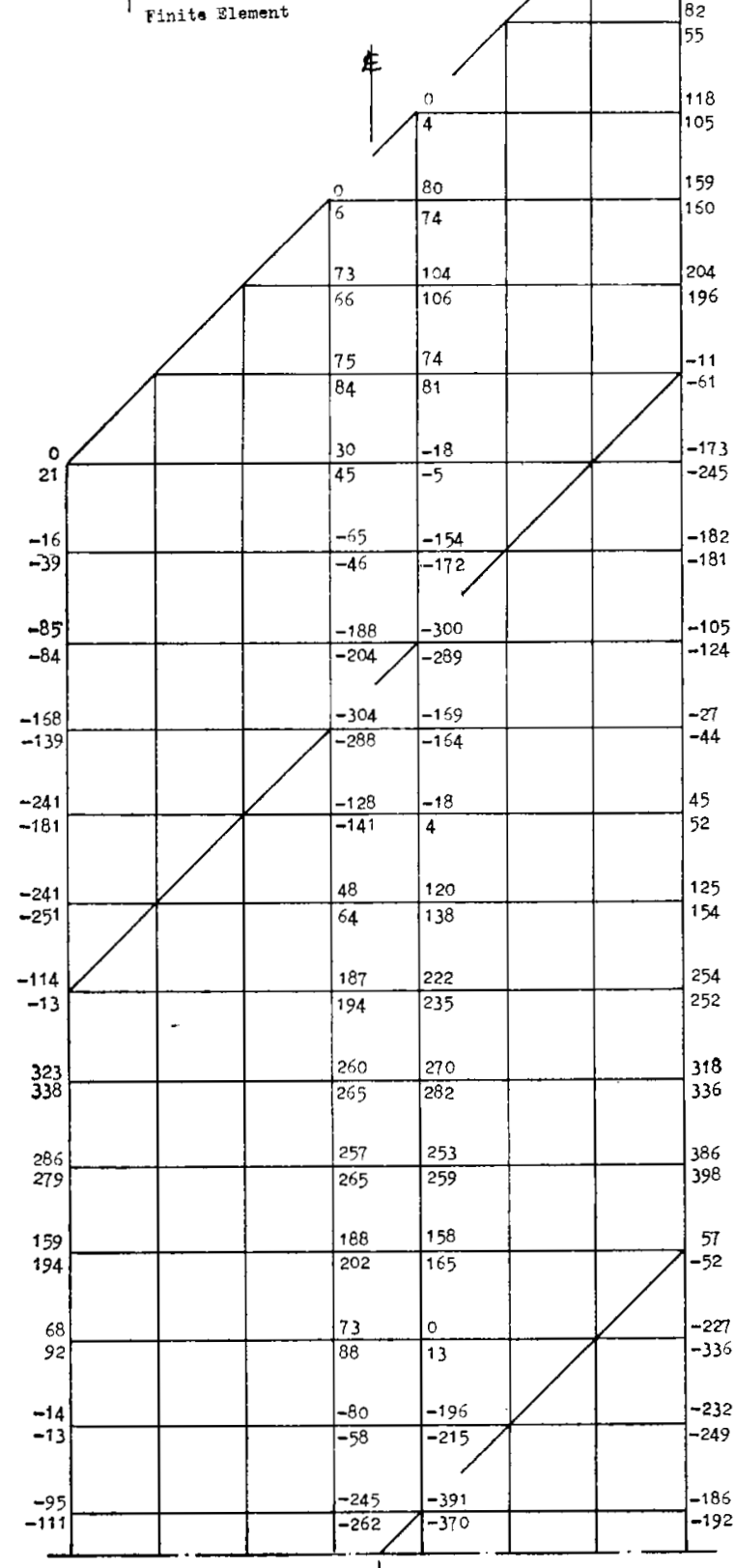

Downloaded by [] on [26/04/23]. Copyright $\odot$ ICE Publishing, all rights reserved. 


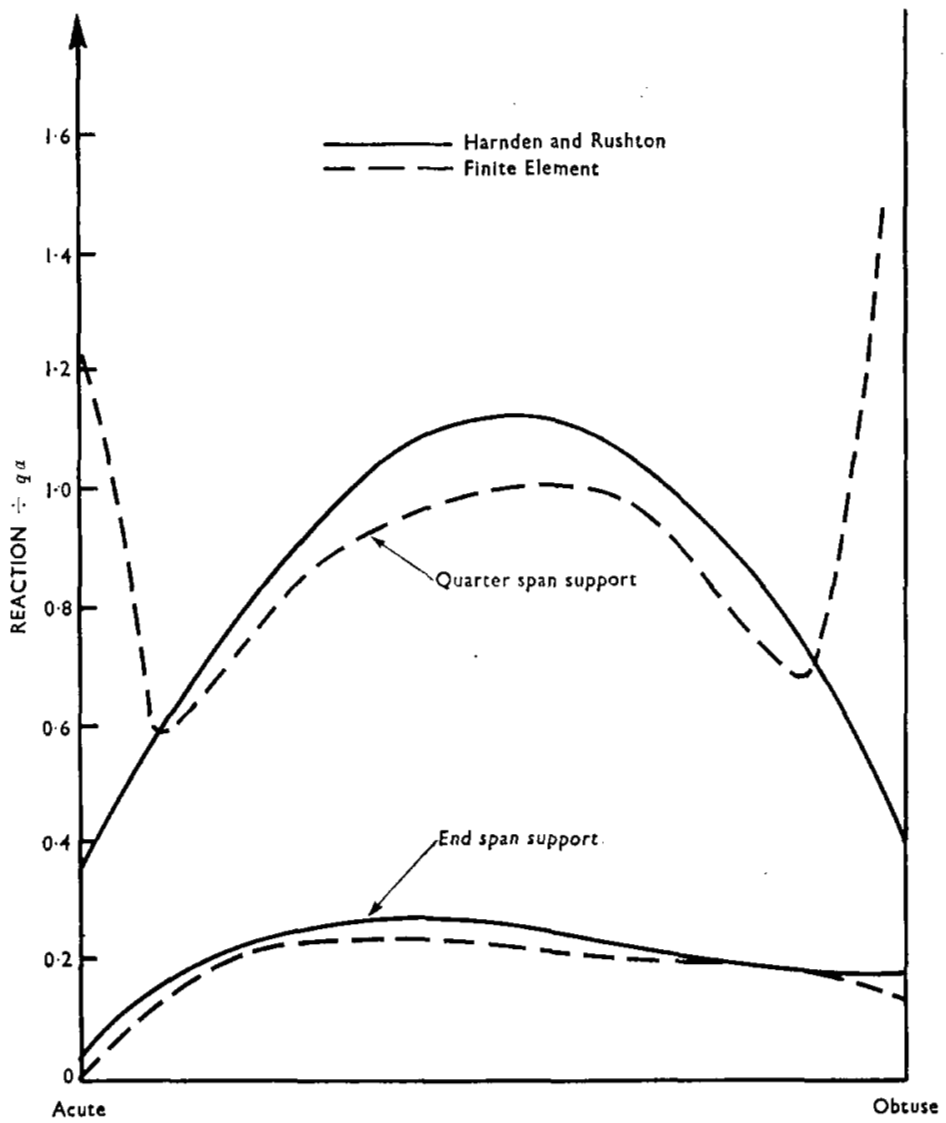

Fig. 23: Distribution OF REACTIONS ON THE END AND QUARTER SPAN SUPPORTS

demonstrated by comparisons with the experimental results of Robinson ${ }^{2}$ and Rusch and Hergenroder. ${ }^{17}$ Common with other finite element solutions, discrete supports and orthotropy do not present any additional complications, since the master program (written in ALGol for the English Electric KDF9 computer) was completely general.

100. To compare a solution obtained using the Writers' approach with the Authors' results, the four span deck was analysed for the uniformly distributed loading case. A line support was taken to coincide with the Authors' structure, and loading was replaced by statically equivalent vertical forces at nodes. Seven elements were taken across the width of the deck, and a square mesh twice as coarse as the one adopted by the Authors was used in the finite element solution.

101. A comparison of deflexions, moments and reactions was given in Figs 19-24. The deflexions agreed well throughout the structure. Moments showed good agreement except at the far ends of the intermediate supports, coinciding with the very severe disagreement in reactions. The finite element solution showed high corner concentrations, and corresponding lower internal values. In an actual bridge 


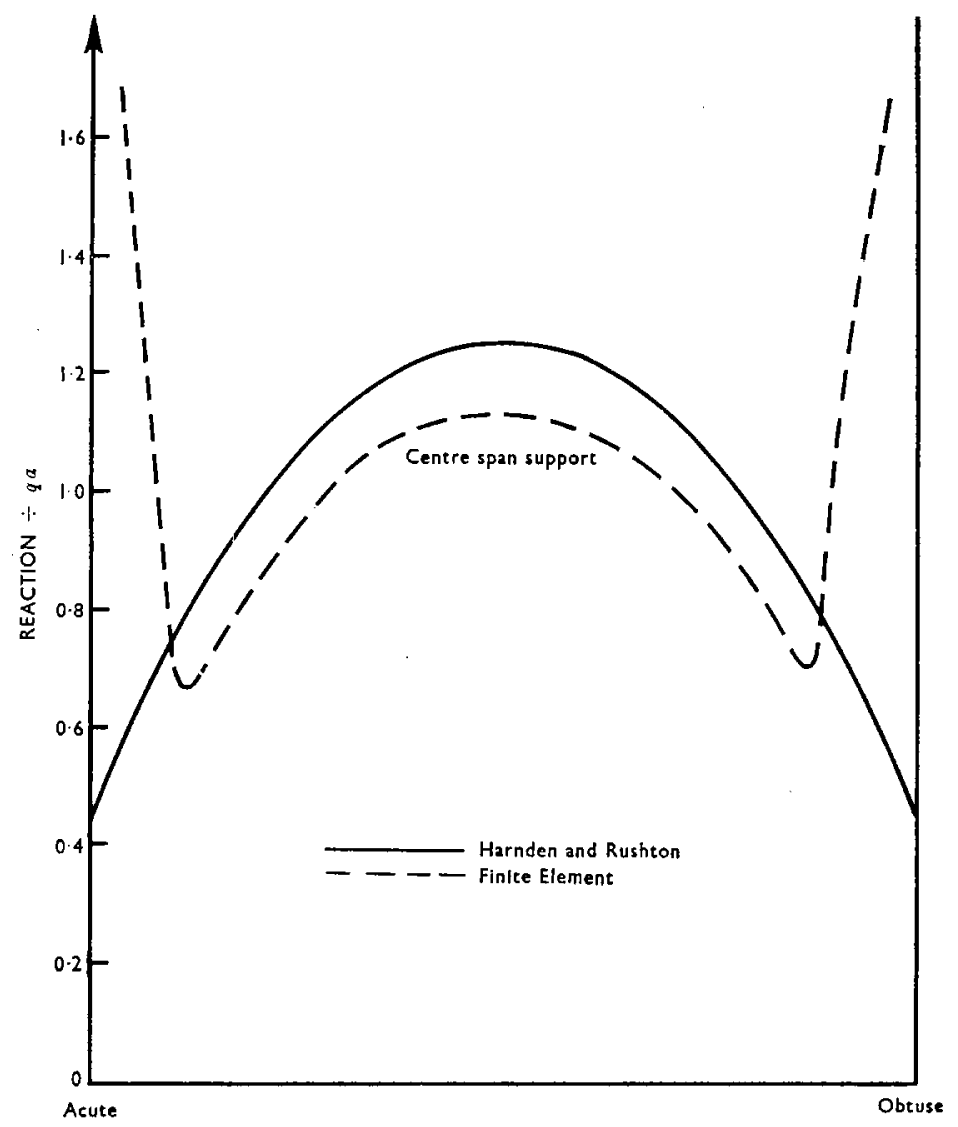

Fig. 24: Distribution OF REACTIONS ON THE CENTRE SUPPORT

structure these high reactions, if present, would tend to re-distribute, but it would be interesting to discover whether they do, in fact, exist.

The Authors, in reply, wished to thank all those who had contributed to the discussion. Different methods of analysing skew bridges had been presented, and it would be left to the reader to assess for himself the relative merits of the methods. A further technique could be added to this list of methods, namely the dynamic relaxation method. Using this technique and following a procedure similar to that reported in reference 18, Otter had solved this skew bridge problem. He kindly lent the Authors a copy of his program and after a slight modification the finite difference solutions on a digital computer using the dynamic relaxation method were found to be identical to the results obtained from the electrical analogue.

103. This led to the most important point in the discussion, the differences found in the distribution of the reactions, Figs 16, 23 and 24 . These differences arose from different assumptions of the boundary conditions at the junction of the free edge and the internal support and the uncertainty in the assumed boundary conditions was 
due to the presence of a mathematical singularity at this corner; this meant that the problem, when stated mathematically, required an infinity in the moments and reactions at this corner. Using a numerical method such as the finite element or finite difference technique, it was not possible to include these infinities, and this lead to the different results.

104. Two approaches were possible when singularities occurred. The first was to modify the numerical technique in the neighbourhood of the singularity so that it was represented correctly; that this could be achieved with finite difference techniques had been demonstrated in reference 19. The second approach was to say that in practice infinitely high moments and reactions could not occur, and then attempt to obtain a good engineering approximation in the neighbourhood of the junction. The second approach was followed by the Authors, they tried different combinations of conditions at the junction of the free edge and internal support (the boundary conditions could be changed without difficulty on the resistance network and the different effects quickly compared). With certain conditions at the junctions, reactions were obtained which tended to become large at the ends of the supports, but in the absence of any reliable experimental evidence it was finally decided that the two conditions to be applied at the junction of the internal supports and free edges should be that the deflexion is zero and that the slope of the bridge is continuous over the support. This gave the deflexions, moments and reactions reported in the Paper.

105. From this discussion, the following conclusions could be made concerning the singularity. First, the singularity had a dominant effect, as illustrated by the differences in Figs 16, 23 and 24, and ignoring the singularity in any problem can lead to serious errors. Secondly, however fine the mesh, the standard numerical methods could not represent the mathematical form of the singularity adequately. This was illustrated by the differences between the finite element results with different mesh spacings, and was discussed further in reference 19. Finally, additional studies into the effect of singularities were clearly required, there was a need at first to include the true effect of the singularity in the numerical technique and then to find what form of stress relaxation takes place in practice. Such studies were being conducted at the University of Birmingham.

106. From their experience of solving the skew bridge problem using both the analogue and the digital computer, the Authors felt able to make the following comparisons. The digital computer program was certainly very powerful, permitting a problem to be stated simply, submitted to the computer, which then produced exhaustive results. With an analogue computer a skilled person was required to set the problem up, and it took him a longer time to obtain the results. However, on the completion of an analogue solution the same set of exhaustive results were available, but in addition the operator had learnt a great deal about the problem, or as an engineer would say, he had got the feel of the problem. He was therefore in a better position to suggest modifications to the design. Unfortunately there was a shortage of engineers able to operate an analogue computer, and for that reason the analogue computer was not used extensively.

107. Finally, in reply to Mr McMillan's questions, the network was deliberately chosen to have a large number of mesh divisions since a comparison between the $64 \times 14$ mesh reported in the Paper, and one with only $32 \times 7$ mesh intervals showed differences of up to $7 \%$ when the bridge was carrying a uniformly distributed load. For a bridge carrying concentrated loads the differences were greater. When it was considered that with the $64 \times 32 \mathrm{mesh}$, each mesh interval represented roughly $2 \mathrm{ft}$, it is clear that, if possible, such a fine mesh should be provided. Mr McMillan also advocated the use of a skew finite difference mesh, and at first it did appear that the skew mesh would be more convenient in fitting the geometry of the problem. However it had been shown ${ }^{4}$ that for a skew mesh the truncation error (the error which arose when the differential equation was written in finite difference form) was larger than for a rectangular mesh, and for skew angles greater than $45^{\circ}$, the skew mesh led to results which were seriously in error. 
REFERENCES

6. ZIENKIEWICZ O. C. et al. Triangular elements in plate bending, conforming and non-conforming solutions. Conf. on Matrix Methods in Struct. Mech. 1965. Wright Patterson Air Base, Ohio.

7. Zienkiewicz O. C. and CheUNG Y. K. The finite element method in structural and continuum mechanics. McGraw-Hill, 1957.

8. JENSEN V. P. Analyses of skew slabs. University of Illinois Bulletin No. 3, 39 1941 (Sept.).

9. MCMillan C. M. The elastic analysis of skew slabs by finite difference methods. M.Sc.(Eng.) Thesis, University of the Witwatersrand, Johannesburg.

10. Asim Yeginobali. Analysis of continuous skewed slab bridge decks. Report No. EES 170-2. 1, 2, 3, Eng. Exp. Station, Ohio State University.

11. HAY J. B. M. An electrical analogy for use in the design of statically indeterminate structures. Selected Engineering Papers No. 146; Institution of Civil Engineers, 1933.

12. Wilby C. B. and Bellamy N. W. Elastic analysis of shells by electronic analogy. Edward Arnold (Publishers) Ltd, 1962.

13. WILBY C. B. A proposed 'exact' theory for analysing shells, and its solution with an analogue computer. Proc. Instn civ. Engrs, 1962, 22 (July) 291-308.

14. Zienkiewicz O. C. and Cheung Y. K. The finite element method for analysis of elastic isotropic and orthotropic slabs. Proc. Instn civ. Engrs, 1964, 28 (Aug.) 471-488.

15. SAwko F. and Cope R. J. The use of finite elements for the analysis of right bridge decks. Int. Symp. on the Use of Computers in Struct. Eng., 1966 (July), Newcastle.

16. SAwko F. and Cope R. J. A new finite element approach to the analysis of skew bridge decks. 1967 (in the press).

17. Rusch $\mathbf{H}$. and Hergenroder A. Influence surfaces for moments in skew slabs (trans. from the German), 1964. Cement and Concrete Association.

18. OTteR J. R. H. Computations for prestressed concrete reactor pressure vessel using dynamic relaxation. Nuclear Structural Engineering, 1965, 1, 61.

19. RUSHTON K. R. The representation of singularities in field problems on an analogue computer. 4th International Analogue Computation Meeting, Brighton 1964, 521-523. 